\title{
Vortex-Loop Unbinding and Flux-Line Lattice Melting in Superconductors
}

\author{
Anh Kiet Nguyen ${ }^{1}$, A. Sudb $\varnothing^{1}$, and R. E. Hetzel ${ }^{2}$ \\ 1 Department of Physics \\ Norwegian University of Science and Technology, N-7034 Trondheim, Norway \\ 2 Department of Theoretical Physics \\ Technical University of Dresden, D-01062 Dresden, Germany
}

\begin{abstract}
We study the interplay between a novel vortex-loop unbinding in finite magnetic field at $T=T_{V}$ and flux-line lattice (FLL) melting at $T=T_{M}$ in type-II superconductors. The FLL melts due to nucleation of vortex loops $\| \hat{c}$-axis, connected to flux lines. For moderate anisotropy, phase coherence $\| \hat{c}$ is lost at $T_{V}>T_{M}$ due to an $a b$-plane vortex-loop unbinding with loops located close to thermal FLL fluctuations. For large anisotropy, phase coherence $\| \hat{c}$ is lost at $T_{V}<T_{M}$ due to nucleation of $a b$-plane vortex-loops uncorrelated to flux lines.
\end{abstract}

PACS numbers: 74.60.-w, 74.60.Ge

It is commonly assumed that the statistical mechanics of the flux-line lattice (FLL) in type-II superconductors is mainly governed by fluctuations of the flux lines around their positions in the ground state Abrikosov FLL, at least at magnetic fields well below the upper critical field such that vortex cores are well separated. Considerable progress in our understanding of the FLL has been made over the last few years, under this assumptiont.

However, we show that for large mass-anisotropies the low-energy thermal excitations in a superconductor in a low magnetic field are quite different. There exists a set of important topological excitations, responsible for destroying longitudinal superconducting phase coherence in a type-II superconductor, which are qualitatively different from fluctuations of flux lines. We also discuss in detail the fluctuations responsible for melting the FLL with no pinning. These two distinct types of fluctuations may influence each other in an essential way. This has observable experimental consequences, as will be discussed. The induction range considered is $0<B / H_{c 2} \ll 1$, which is known to be the relevant limit for considering the phenomenon of FLL melting in extreme type-II superconductors with uniaxial anisotropy of the superconducting order parameter are unimportant, and the anisotropic London model is appropriatet.

The model we consider is a discretized, uniaxial anisotropic superconductor in the London limit $\bar{E}$, on a lattice with $L_{\perp}^{2} \times L_{z}$ in units of the numerical lattice constant $d$. The anisotropy is along the crystal $\hat{c}$-axis, described by the parameter $\Gamma=\lambda_{c} / \lambda_{a}=\sqrt{M_{z} / M}$, where $\lambda_{a}$ and $\lambda_{c}$ are the magnetic penetration lengths along the crystal $a b$-plan and the crystal $\hat{c}$-axis, respectively, and $M_{z}, M$ are normal-state quasiparticle effective masses along the $z$-direction and $a b$-planes, respectively. We take our coordinate system $(\hat{x}, \hat{y}, \hat{z})$-axes parallel to the crystal $\hat{a}, \hat{b}$ and $\hat{c}$-axes, respectively. Periodic boundary conditions inall directions are used. The Hamiltonian is given by 4 6

$$
H=2 \pi^{2} J_{0} \sum_{i, j} \sum_{\mu=x, y, z} G_{\mu}\left(\mathbf{r}_{i}-\mathbf{r}_{j}\right) n_{\mu}\left(\mathbf{r}_{i}\right) n_{\mu}\left(\mathbf{r}_{j}\right),
$$

where $n_{\mu}\left(\mathbf{r}_{i}\right)$ is the integer vorticity through plaquette $\mu$ at site $\mathbf{r}_{i}$. Note that this model allows for arbitrary fluxline shapes, and also incorporates topological vortex loop excitations. $J_{0}=\Phi_{0}^{2} d / 16 \pi^{3} \lambda_{a}^{2}$ and $J_{0} / \Gamma^{2}$ are the energy scales for excitations involving $n_{z}(\mathbf{r})$ and $\left(n_{x}(\mathbf{r}), n_{y}(\mathbf{r})\right)$, respectively, and $\Phi_{0}$ is the flux quantum. $G(\mathbf{r})$ is the lattice London interaction, with Fourier transform

$$
\begin{aligned}
G_{x, y}(\mathbf{k}) & =\frac{\Gamma^{-2}}{Q_{x}^{2}+Q_{y}^{2}+\Gamma^{-2}\left(Q_{z}^{2}+d^{2} \lambda_{a}^{-2}\right)} \\
G_{z}(\mathbf{k}) & =\frac{\left(Q^{2}+d^{2} \lambda_{c}^{-2}\right) /\left(Q^{2}+d^{2} \lambda_{a}^{-2}\right)}{Q_{x}^{2}+Q_{y}^{2}+\Gamma^{-2}\left(Q_{z}^{2}+d^{2} \lambda_{a}^{-2}\right)},
\end{aligned}
$$

where $Q_{\mu}=2 \sin \left(k_{\mu} d / 2\right), Q^{2}=\sum_{\mu} Q_{\mu}^{2}$. In these simulations, we have chosen $\lambda_{a}=0.75 \bar{a}$, where $\bar{a}$ is the average distance between flux lines.

To probe superconducting phase coherence, we consider the helicity modulus $\Upsilon_{\mu}\left(q_{\nu}\right)(\mu \neq \nu)$. For a given perturbation in the external vector potential $\delta A_{\mu}^{e x t}\left(q_{\nu}\right) \hat{\mu}$, $\Upsilon_{\mu}\left(q_{\nu}\right)$ is the linear response coefficient giving the induced supercurrent $\mathbf{j}$

$$
j_{\mu}\left(q_{\nu}\right)=-\Upsilon_{\mu}\left(q_{\nu}, T\right) \delta A_{\mu}^{e x t}\left(q_{\nu}\right) .
$$

We find that the helicity modulus in a uniaxial anisotropic lattice superconductor is given by

$$
\frac{\Upsilon_{\mu}\left(q_{\nu}, T\right)}{\Upsilon_{\mu}\left(q_{\nu}, 0\right)}=1-\frac{4 \pi^{2} J_{0} \lambda_{a}^{2}}{V T} \frac{<n_{\sigma}\left(q_{\nu}\right) n_{\sigma}\left(-q_{\nu}\right)>}{1+\left(1+\delta_{\mu, z}\left(\Gamma^{2}-1\right)\right) \lambda_{a}^{2} Q^{2}},
$$

where $<. .>$ denotes a thermal average, $(\mu, \nu, \sigma)$ are cyclic permutation of $(x, y, z)$, and $\mathrm{V}$ is the volume of the lattice.

We investigate the melting of the FLL by considering the structure factor for $n_{z}$ vortex elements 


$$
S(\mathbf{k})=\frac{<\left|\sum_{i} n_{z}\left(\mathbf{r}_{i}\right) \exp \left[i \mathbf{k} \cdot \mathbf{r}_{i}\right]\right|^{2}>}{N_{z}^{2}},
$$

where $N_{z}=\sum_{i} n_{z}\left(\mathbf{r}_{i}\right)$. In the ground state the FLL has the well know hexagonal form when $\mathbf{B} \| \hat{c}$, and $S\left(\mathbf{K}, k_{z}=\right.$ $0)$ has $\delta$-function Bragg peaks at the reciprocal lattice points $\mathbf{K}$. When the FLL melts, the Bragg peaks are washed out. The lowest $T$ where $S(\mathbf{K})$ vanishes thus defines the melting temperature $T_{M}$.

We employ the following Monte Carlo (MC) procedure: We start with fixed average density $\mathrm{f}=\mathrm{B} / \Phi_{0}$ of straight vortex lines parallel to the $\hat{z}$-axis. We update the system, heating from the ground state by sweeping over the lattice in a systematic way. For each site we try to add one elementary closed vortex loop, choosing one of six possible orientations of the vortex loop at random. These moves are accepted or rejected according to the standard Metropolis algorithm. This MC procedure provides a complete sampling of the phase space of the vortex variables $\mathbf{n}(\mathbf{r})$, subject to two constraints: 1) $\triangle \cdot \mathbf{B}(\mathbf{r})=0$, where $\mathbf{B}(\mathbf{r})$ is the local induction and $\triangle$ is the lattice gradient operator. 2) The average induction is determined by $\mathbf{B}=\left(\Phi_{0} / V\right) \sum_{i} \mathbf{n}\left(\mathbf{r}_{i}\right)=f \Phi_{0} \hat{z}$ is constant. Note that our MC procedure allows for excitations of free vortex loops, i.e vortex loops not connected to any existing vortex lines. Each data point is obtained after discarding the first 5000 steps to allow for equilibration, whereas the subsequent 10000-40000 steps are used to obtain averages, where each step refers to a sweep through the entire $L_{\perp}^{2} \times L_{z}$ lattice.

In these simulations, we use $f=1 / 48, L_{\perp}=24$ and $L_{z}=12$. The reciprocal lattice vector of the vortex lattice $\mathbf{K}$ is taken to be the lowest in $K_{x}$-direction consistent with the filling fraction $f=1 / 48$ and a square numerical mesh, i.e. $\mathbf{K}=(4,0) 2 \pi / L_{\perp}$. The $q$-value chosen in Eq. 4 is the lowest finite one on our numerical lattice, i.e. $\mathbf{q}_{x}=(1,0) 2 \pi / L_{\perp}$ and $\mathbf{q}_{y}=(0,1) 2 \pi / L_{\perp}$.

$\Upsilon_{x, y}$ will always be zero in finite field when the FLL is unpinned, since the FLL will move in response to an arbitrarily weak applied current. In our lattice model, the numerical lattice introduces artificial pinning which we must eliminate in order to be able to study the true melting of the FLL. This is achieved for low enough values of $f<f_{c}$, where we have found $f_{c} \approx 1 / 32$ for the isotropic systems and decreasing very slightly with increasing mass-anisotropy. It suffices that $\Upsilon_{x, y}$ vanishes at temperatures below those of which $S(\mathbf{K})$ vanishes. This indicates that the FLL 'floats' freely on the numerical lattice, having thermally depinned from it, in the form of an intact FLL as demonstrated by a finite $S(\mathbf{K})$. This allows a study of the actual FLL-melting without artificial pinning effects 8 . As shown in both Figs. 1 and 2 , this is clearly so in our simulations. We have also checked that the $T$-dependence of $\Upsilon_{z}$, practically is not influenced by $f$ if $f<1 / 16$.

The quantitity $\Upsilon_{z}(q)$ essentially measures phasecoherence along the $\hat{z}$-axis. Since vortex loops need not be connected to flux lines, $\Upsilon_{z}(q)$ may vanish without a corresponding vanishing of $S(\mathbf{K})$. Thermal fluctuations may destroy superconducting phase coherence while leaving the FLL intact. This could possibly be interpreted as the finite-field counterpart of the well known 'inverted XY-transition' found in a $3 D$ lattice superconductor model in zero magnetic field 3 .

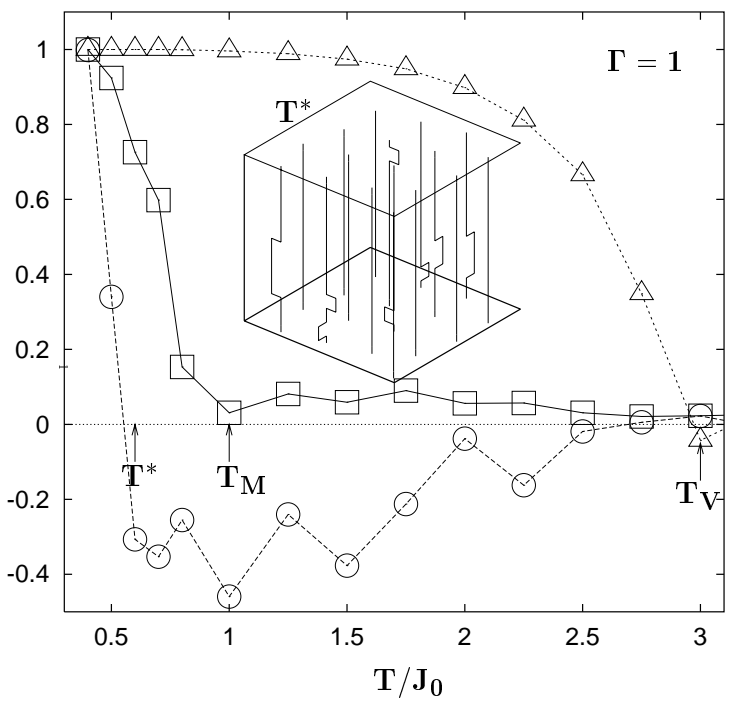

FIG. 1. $\Upsilon_{z}\left(q_{x}, T\right) / \Upsilon_{z}\left(q_{x}, 0\right)(\triangle), \Upsilon_{x}\left(q_{y}, T\right) / \Upsilon_{x}\left(q_{y}, 0\right)(\bigcirc)$, and $S(\mathbf{K})(\square)$ as a function of temperature for $\Gamma=1$. Superconducting phase-coherence along the direction of the flux lines is left intact in the flux-line liquid $S(\mathbf{K})=0$, indicating a melting transition into a disentangled vortex liquid. Shown in the inset is a snapshot of the flux-line system at $T^{*}<T_{M}$. All excitations are thermally nucleated flux-line defects. There are no free vortex loops in the system, as explained in text. Note how $\Upsilon_{x}$ vanishes before $S(\mathbf{K})$; the system is in a 'floating solid phase'.

In Fig. 1, $S(\mathbf{K}), \Upsilon_{x}$ and $\Upsilon_{z}$ are shown as functions of the temperature for $\Gamma=1$. The helicity modulus $\Upsilon_{z}$ is seen to vanish at considerably higher temperatures than $S(\mathbf{K})$. Moreover, $\Upsilon_{x}$ vanishes at a temperature distinctly below $T_{M}$, such that by the time we reach the FLL melting temperature $T=T_{M}$, we have established a 'floating solid phase' from which the FLL can melt with no artificial pinning effects to the numerical lattice. The fact that $\Upsilon_{z}$ stays finite through the melting transition where $S(\mathbf{K})$ vanishes, indicates that the flux lines stay intact in the vortex liquid, without large defects as evidenced by the still finite $\Upsilon_{z} . S(\mathbf{K})$ vanishes due to loss of shear stiffness between flux lines with large effective tilt-moduli. As the temperature is increased further, $\Upsilon_{z}$ eventually vanishes also, at $T=T_{V}$. For the isotropic case, this is primarily due to fluctuations in the flux lines, or equivalently directed vortex loops $\| \hat{z}$-axis attached to flux lines. This is easily understood, since vortex loops $\| \hat{z}$-axis and vortex loops in the $a b$-plane have the same self-energy when $\Gamma=1$, but vortex loops $\| \hat{z}$-axis attached to flux lines gain energy by annihilating one segment of the loop 
with one segment of a flux line. Moreover, when vortex loops parallell to the $a b$-planes eventually are nucleated for $\Gamma \lesssim 3$, they tend to be nucleated in close vicinity to flux-line defects; they may interact attractively with the non-vertical (here horizontal) components of these defects.

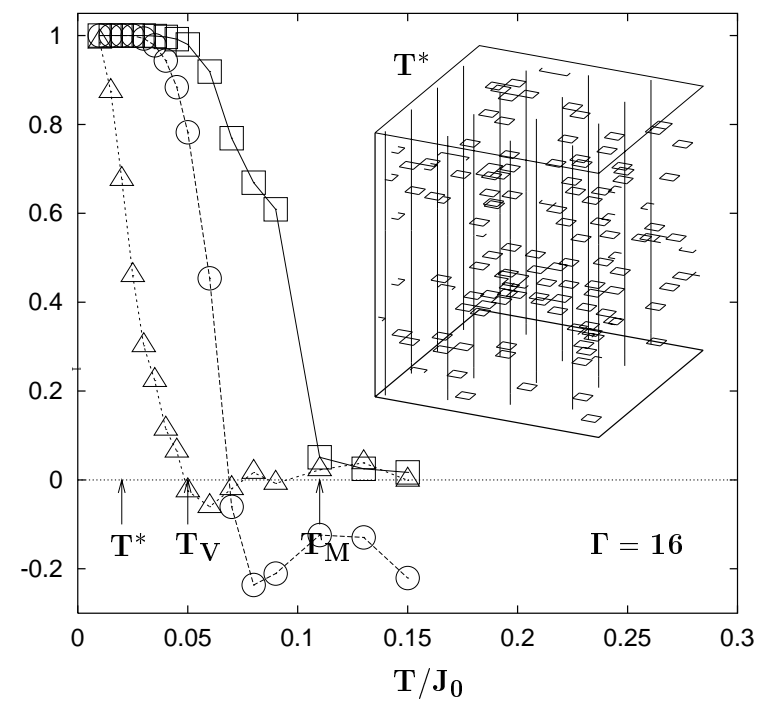

FIG. 2. $\Upsilon_{z}\left(q_{x}, T\right) / \Upsilon_{z}\left(q_{x}, 0\right)(\triangle), \Upsilon_{x}\left(q_{y}, T\right) / \Upsilon_{x}\left(q_{y}, 0\right)(\bigcirc)$, and $S(\mathbf{K})(\square)$ as a function of temperature for $\Gamma=16$. Superconducting phase-coherence along the direction of the flux lines is lost before the FLL melts. Shown in the inset is a snapshot of the flux-line system at $T^{*}<T_{M}$. Note the proliferation of $a b$-plane vortex loops uncorrelated to flux lines; the FLL is left intact.

When $\Gamma>5$, vortex loops in the $a b$-planes are easily nucleated anywhere in the $a b$-plane, due to the large reduction of theself-energy of flux lines per unit length, by a factor $1 / \Gamma^{211}$. The result is a dramatic enhancement of vortex-loop unbinding and a resulting reduction of $T_{V}$ at which $\Upsilon_{z}(q)$ vanishes. This is shown in Fig. 2, where it is seen that, for $\Gamma=16, \Upsilon_{z}$ vanishes at temperatures well below the temperature $T_{M}$ where $S(\mathbf{K})$ vanishes. $\Upsilon_{z}$ vanishes, not due to fluctuations of the flux lines along the $\hat{z}$-axis, but due to nucleation of vortex loops in the $a b$ planes located between flux lines. We conjecture that this transition very likely may be a finite-field counterpart of the 'inverted XY transition' first discussed by Dasgupta and Halperin of a lattice superconductor in zero-field 8 . Flux-line defects are nucleated only at much higher temperatures, and the FLL is left completely intact at the vortex-loop unbinding transition, as shown by the value of $S(\mathbf{K})$ when $\Upsilon_{z}$ vanishes. This is also clearly illustrated in the inset of Fig. 22.

Vortex-loops $\| \hat{z}$-axis also become easier to nucleate when $\Gamma$ increases, but only by half the amount of the $a b$-plane loops, since an elementary loop $\| \hat{z}$-axis has two, and not four, flux-line segments parallell to the $a b$-plane. Moreover, it is always energetically favourable to attach them to already existing flux lines, by the same argument as in the isotropic case. Hence, flux-line defects are also easier to nucleate in the anisotropic case, as one would have guessed from the reduction of the nonlocal tilt-modulus of the FLL when $\Gamma$ increases 3 . Practically no vortex loops $\| \hat{z}$-axis are nucleated away from existing flux lines for $T \leq T_{M}$.

For sufficiently large $\Gamma, \Upsilon_{z}$ vanishes at temperatures well below those at which $S(\mathbf{K})$ vanishes, i.e. $T_{V} \ll T_{M}$. Nonetheless, the melting of the FLL is not necessarily $2 D . T_{M}(\Gamma)$ continues to drop for increasing values of $\Gamma$ which are much larger than the value of $\Gamma$ where $T_{V}=T_{M}$. Thus, even if $T_{V} \ll T_{M}, T_{M}$ still depends on the coupling between planes. As long as this is the case, the FLL melting transition is therefore $3 D$ in character. This is shown in Fig. 3, where both $T_{V}$ and $T_{M}$ are plotted versus $\Gamma$. $T_{V}=T_{M}$ for $\Gamma \approx 5$. However, $T_{M}$ oply saturates to a finite value (which implies $2 D$ melting 12 ) at a much larger value, $\Gamma \approx 16$. We have found an intermediate range of $\Gamma$-values where the entropy jump $\Delta S$ associated with the FLL melting increases with 110.

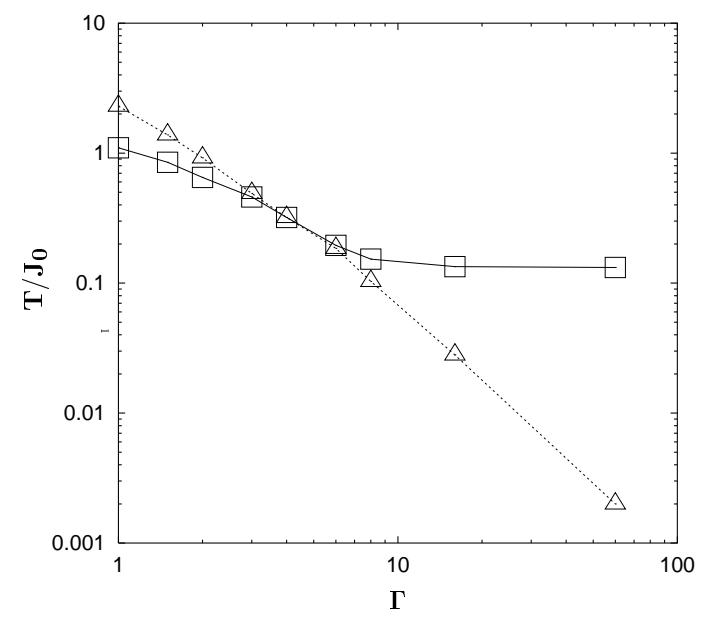

FIG. 3. Melting temperature $T_{M}(\square)$ and vortex-loop unbinding temperature $T_{V}(\triangle)$ as a function of $\Gamma$. Note saturation at $\Gamma>16$, showing that the melting eventually becomes $2 D$, but only for considerably larger values of $\Gamma$ than those values where $T_{M}=T_{V}$.

Previously, an entropy jump of $\Delta S \bar{\square} 0.3 k_{B}$ per vortex per layer was found theoretically well in agreement with a number of experiments 1415 . We now propose a picture for the variation $\Delta S$ with $\Gamma$ and induction $B$, when the perfect Abrikosov FLL has been gauged away by a singular gauge-transformation 16 . Hence, only thermally nucleated vortex-loops and thermally nucleated flux-line defects are relevant degrees of freedom, precisely as in our Monte Carlo simulations.

The character of the vortex-loop unbinding and FLL melting transitions is governed by the effective interactions between vortex-loops in the $a b$-planes and the interaction between flux-line defects, respectively. The effective interaction between vortex loops is screened by ther- 
mally nucleated flux-line defects, but is left unscreenged by the flux lines in the rigid Abrikosov vortex latticelle.6. Conversely, flux-line defects are screened by vortex loops. Note that this is different from the electromagnetic screening of the interaction between field-induced vortices, even in the perfect Abrikosov FLL. Such screening is present at all temperatures.

Vortex-loop unbinding transition: An unscreened interaction between vortex loopstate makes the vortexloop unbinding transition continuous, while screening will tend to drive it first order. Increasing $\Gamma$ will enhance thermal nucleation of flux-line defects, as argued above, enhancing screening of the vortex-loop interaction, driving the vortex-loop unbinding more first order. If one increases $B$, an increase in the density of flux-line defects will also enhance screening of the effective interaction between vortex loops, enhancing the first order character of the transition. However, it will also suppress fluctuations in individual flux lines, by increasing the flux-line tilt modulus $c_{44}$. At low $B c_{44} \sim B^{2}$, while the areal density of flux-lines $n \sim B$. Therefore, the dominant effect is the increase of the areal density of flux-line defects. Screening of the vortex-loop interaction is increased, driving the vortex-loop unbinding more discontinuous. There will be a crossover field $B^{*}$ above which the increase in $c_{44}$ dominates, which will then reduce the number of flux-line defects. Above this field, the vortex-loop unbinding transition will become less first order.

FLL melting transition: An unscreened interaction between flux-line defects will make the FLL melting transition continuous. Screening of this interaction will tend to drive it first order. Increasing $\Gamma$ will increase the nucleation of vortex loops in the $a b$-plane, enhancing the screening of the interaction between flux-line defects, driving the melting of the FLL more first order. On the other hand, if the magnetic field increases, it is clear that the interaction between thermally nucleated flux-line defects will be less screened, driving the transition more continuous 17. The FLL melting transition thus becomes less first order in the anisotropic case as $B$ increases, due to the presence of already thermally nucleated $a b$-plane vortex loops. Therefore the entropy jump associated with the transition is reduced as $B$ increases.

Recently, an interesting decrease in the entropy jump $\Delta$ S with increasing $B$ was in fact observed by Zeldov et al. . 5 . Note that a consideration of fluctuations of the flux lines only, will give $\Delta S \sim B^{1 / 2}$, an opposite trend 5 . The above discussion shows that inclusion of vortex-loops into the consideration of the FLL melting in a natural way explains the data of Zeldov et al.. The vortex-loop unbinding is crucial for the statistical mechanics of a type-II superconductor in magnetic fields $B \in(0-2 T)$.

We have discussed the existence of, and interplay between, two distinct phase-transitions in type-II superconductors. One is the melting of the FLL, which looses its meaning as the induction goes to zero. The other is a novel vortex-loop unbinding transition in finite magnetic field, with a zero-field counterpart. We emphasize two novel aspects of this work. The vortex-loop unbinding transition has been observed i) in a finite magnetic field and ii) both in the flux-line lattice and flux-line liquid cases, depending on our choice of $\Gamma$.

Support from the Research Council of Norway under Grants No. 110566/410 and 110569/410, is gratefully acknowledged. We thank K. Fossheim, F. Kusmartsev, Z. Tešanović, and J. M. Wheatley for discussions, and F. Mo for use of computational facilities.

${ }^{1}$ G. Blatter, et al., Rev. Mod. Phys., 66, 1125 (1994); E. H. Brandt, Rep. Prog. Phys. 58, 1465 (1995).

${ }^{2}$ D. R. Nelson and H. S. Seung, Phys. Rev. B 39, 9153 (1989).

${ }^{3}$ A. Houghton, R. A. Pelcovits, and A. Sudbø, Phys. Rev. B 40, 6763 (1989); E. H. Brandt, Phys. Rev. Lett., 63, 1106 (1989).

${ }^{4}$ A. Sudbø and E. H. Brandt, Phys. Rev. B 43, 10482 (1991).

${ }^{5}$ R. Cavalcanti, G. Carneiro, and A. Gartner, Europhys. Lett. 17, 449 (1992); R. Cavalcanti, G. Carneiro, and A. Gartner, Phys. Rev. B 47, 5263 (1993).

${ }^{6}$ G. Carneiro, Phys. Rev. B 45, 2391 (1992); ibid B 45, 2403 (1992); ibid B 50, 6982 (1994).

7 T. Chen and S. Teitel, Phys. Rev. Lett. 74, 2792 (1995).

${ }^{8}$ M. Franz and S. Teitel, Phys. Rev. Lett., 73, 480, (1994); S. Hattel and J. M. Wheatley, Phys. Rev. B 50, 16590 (1994); ibid B 51, 11951 (1995).

${ }^{9}$ C. Dasgupta and B. I. Halperin, Phys. Rev. Lett., 47, 1556 (1981); W. Janke and T. Matsui, Phys. Rev. B 42, 10673 (1990); S. E. Korshunov, Europhys. Lett., 11, 757 (1990); B. Chattopadhyay and S. R. Shenoy, Phys. Rev. Lett., 72, 400 (1994); G. Williams, Phys. Rev. Lett., 59, 1926 (1987).

${ }^{10}$ Calculations of the entropy discontinuity both at the vortex-loop unbinding transition and the FLL melting transition, as a function of $\Gamma$, are in progress. In this paper $L_{z}=12$. Simulations for $L_{z}=6,12,18,24$ reveal that for $\Gamma=1$, the transitions become sharper, while for $\Gamma=16$ the transitions are unaffected by increasing $L_{z}$.

11 A. Sudb $\varnothing$ and E. H. Brandt, Phys. Rev. Lett., 66, 1781 (1991).

12 S. Doniach and B. Hubermann, Phys. Rev. Lett. 42, 1169 (1979); D. S. Fisher, Phys. Rev. B 22, 1190 (1980).

13 R. E. Hetzel, A. Sudbø, and D. A. Huse, Phys. Rev. Lett., 69, 518 (1992).

${ }^{14}$ H. Pastoriza et al., Phys. Rev. Lett., 72, 2951 (1994); R. Liang, D. A. Bonn, and W. N. Hardy, Phys. Rev. Lett., 76, 835 (1996).

${ }^{15}$ E. Zeldov et al., Nature, 375, 373 (1995).

16 Zlatko Tešanović, Phys. Rev. B 51, 16204 (1995).

${ }^{17}$ We thank Z. Tešanović for discussions on this point. 